\title{
CONDUCTION BAND INFLUENCE ON THE PROPERTIES OF BISTABLE DONORS*
}

\author{
S. BEDNAREK AND J. ADAMOWSKI \\ Institute of Physics and Nuclear Techniques, Academy of Mining and Metallurgy \\ Al. Mickiewicza 30, 30-059 Kraków, Poland
}

\begin{abstract}
A theoretical description of bistable donors in polar semiconductors is proposed. The donor states are described within the one-band approximation, which takes into account a finite width and nonparabolicity of the conduction band. The interaction between the defect and the crystal lattice is assumed in the Fröhlich form. For the bistable indium impurity in cadmium fluoride, a coexistence of strongly and weakly localized donor states has been obtained. The calculated energies for both the states and absorption band shape in the $3-\mathrm{eV}$ range are in agreement with experiment.
\end{abstract}

PACS numbers: 71.55.-i

Optical absorption spectra of cadmium fluoride crystals doped with indium exhibit unusual properties [1, 2, 3]. In these crystals, the two asymmetric absorption bands are observed $[2,3]$ with the maxima at $0.2 \mathrm{eV}$ and $3 \mathrm{eV}$, respectively. The sample cooled in darkness shows in low temperatures the $3-\mathrm{eV}$ band only, while the sample illuminated with the white light starts to absorb the infrared (IR) radiation in the $0.2-\mathrm{eV}$ band. During the illumination, the IR absorption increases, while the absorption in the visible and ultraviolet range decreases. The threshold for the visible absorption is determined to be $1.9 \mathrm{eV}$ [2] and the thermal activation energy for the Hall effect is $0.25 \mathrm{eV}$ [2]. The difference between these values, i.e. $1.65 \mathrm{eV}$, is the Stokes shift. These results lead to the conclusion [2, 3] that the indium impurity in $\mathrm{CdF}_{2}$ is a bistable donor which possesses two sets of quantum states with energy levels in a gap: strongly localized states, which are responsible for the absorption in the $3 \mathrm{-eV}$ range, and weakly localized shallow states, which are responsible for the absorption in the $0.2-\mathrm{eV}$ range. This interpretation has been confirmed by the results of theoretical investigations $[4,5]$. In the present paper, the theory $[4,5]$ is extended by taking into account a finite width of the conduction band, performing all the calculations in the $k$-space with the Debye

*This work is supported in part by the Ministry of National Education under the contract DSN-P/04/368/90-2. 
cut-off and introducing the variational basis with arbitrary number of elements. This approach is applied to a description of the 3-eV absorption band shape.

We consider the system which consists of the donor center, the electron and the crystal lattice. The interaction of the donor center and the electron with the lattice is taken on as the polar (Fröhlich) coupling and other couplings with the lattice are neglected. The donor-electron interaction is assumed to be coulombic. We as well take into account a short-range potential which is constant in the $k$-space and changes the energy of strongly localized states. The present approach goes beyond the effective mass approximation and is based on the one-band approximation. The dispersion relation for the conduction band is proposed in the form

$$
E_{\mathrm{c}}(k)=a\left(k / k_{0}\right)^{2}+b\left\{1-\left[1+\left(k / k_{0}\right)^{2}\right]^{-2}\right\} .
$$

This formula contains one fitting parameter $k_{0}$; the values of $a$ and $b$ are determined from the known electron band edge mass and conduction band width [6]. The eigenvalue problem for the donor is solved with the help of a variational method in the $k$-space. The donor envelope is proposed as a linear combination of the terms

$$
\phi_{i}(k)=N_{i}\left(k^{2}+\beta_{i}^{2}\right)^{-2},
$$

where $N_{i}$ are the normalization constants and $\beta_{i}$ are the variational parameters. The calculations have been performed with 1- and 3-element basis (2). The 1-element basis is quite sufficient for shallow donor states, however, the use of 3 -element basis improves the results for the localized donor state. The summation over the first Brillouin zone is approximated by the integration over the Debye sphere in the $k$-space.

This approach has been applied to a description of the bistable indium donor in $\mathrm{CdF}_{2}$. The results of calculations are shown in Fig. 1, in which the expectation value $E$ of the Hamiltonian for the donor-lattice system is plotted against the localization parameter $\lambda$ for the donor state defined as the expectation value of the inverse electron-donor separation, i.e., $\lambda=\langle 1 / r\rangle$. The curve $E(\lambda)$ possesses two minima: the local minimum for the smaller localization $\lambda_{1}$ and the global minimum for the larger localization $\lambda_{2}$. The calculated energy $E\left(\lambda_{1}\right)$ is in agreement with the measured $[2,3]$ shallow donor energy. However, the experimental value $[2,3]$ for the strongly localized state is correctly reproduced with the use of 3-element basis (2). Then, the estimated localization is slightly smaller than $\lambda_{2}$. The maximum of the $E(\lambda)$ corresponds to an energy barrier obtained in experiment [1, 2]. The calculated overlap between the wave functions of the donor-lattice system, which correspond to the different localizations, is negligibly small [4]; therefore, we get two orthogonal quantum states. Thus, the coexistence of strongly and weakly localized donor states has been theoretically proved in agreement with experiment $[2,3]$.

The absorption in the 3-eV range results from the Franck-Condon transitions between the strongly localized donor states (initial states) and the delocalized conduction band states (final states). In the initial state, the crystal lattice is relaxed, 


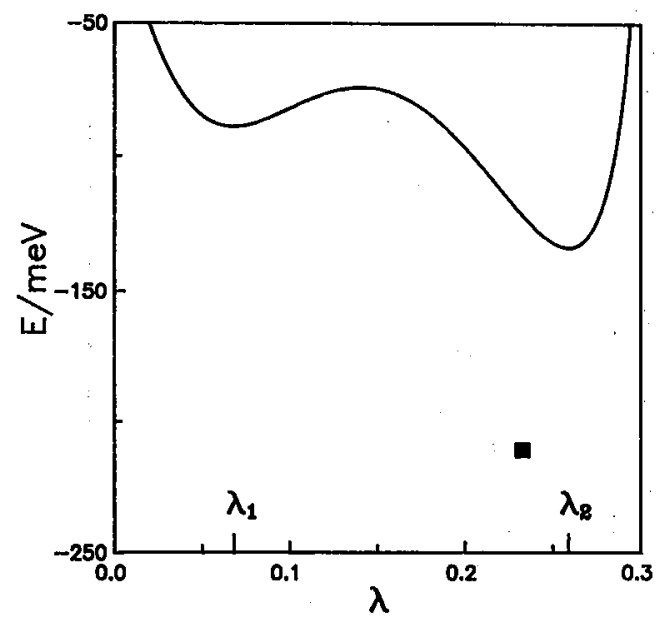

Fig. 1. Expectation value $E$ of the Hamiltonian for the donor-lattice system as a function of the localization parameter $\lambda$, which is defined as the average value of the electron-donor distance, i.e., $\lambda=\langle 1 / r\rangle$, and measured in inverse Bohr radii. The solid curve shows the results obtained with the 1-element basis, the square denotes the result for the strongly localized state obtained with the 3-element basis. Energy is measured with respect to the conduction band minimum.

i.e., its quantum state corresponds to a minimum energy of the donor-lattice system. If the optical absorption is caused by the Franck-Condon transitions, the system goes over into the state with nonrelaxed lattice, i.e., the lattice configuration does not change and remains the same as in the initial state. We have calculated the transition probabilities for the absorption to the nonrelaxed states using a method, which will be described elsewhere [7]. Figure 2 shows the calculated absorption band shape in the 3-eV range. An agreement with experiment [2, $3]$ is fairly good. Because the absorption coefficient contains information about the conduction band shape, this agreement confirms the validity of parametrization (1) proposed for the conduction band.

The results of the present paper allow us to clarify the nature of the bistability of indium donors in $\mathrm{CdF}_{2}$. This bistability is caused by an interplay between the electronic and lattice properties of the defect. The electronic properties, which are most important for the bistability, are the finite width and nonparabolicity of the conduction band. The lattice influence is manifested through the donor-lattice polar coupling. The incorporation of the short-range donor potential results in a shift of the energy level of the localized state and does not change the qualitative properties of the system. 


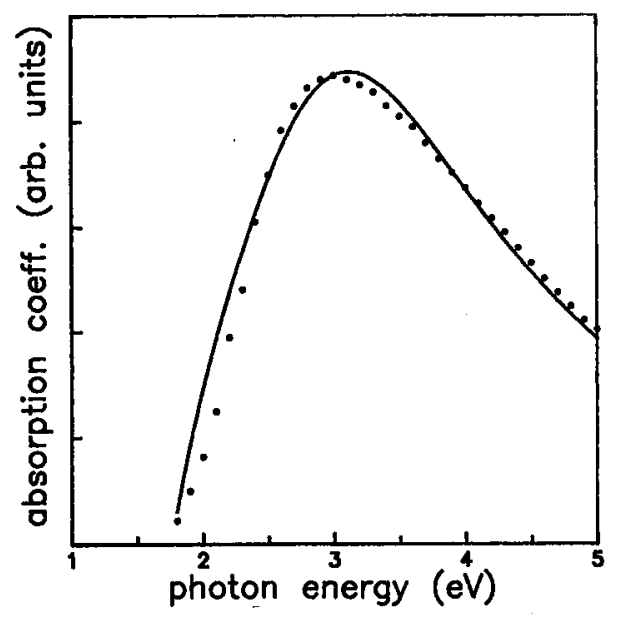

Fig. 2. Calculated (solid) and measured (dotted) absorption coefficient versus the absorbed photon energy.

\section{References}

[1] J.M. Langer, J. Phys. Soc. Jpn. 49, Suppl. A, 207 (1980).

[2] J.E. Dmochowski, J.M. Langer, Z. Kalinski, W. Jantsch, Phys. Rev. Lett. 56, 1735 (1986).

[3] J.E. Dmochowski, J.M. Langer, W. Jantsch, in Shallow Impurities in Semiconductors 1988, Ed. B. Monemar, Institute of Physics Conference Series No. 95, Bristol and Philadelphia 1989, p. 325.

[4] S. Bednarek, Acta Phys. Pol. A77, 115 (1990).

[5] S. Bednarek, J. Adamowski, Materials Science Forum 65-66, 427 (1990).

[6] J.P. Albert, C. Jouanin, C. Gout, Phys. Rev. B 16, 4619 (1977).

[7] S. Bednarek, to be published. 